\title{
Long-term follow-up of stereotactic radiosurgery for head and neck malignancies
}

\author{
Dawn Owen, MD, PhD, ${ }^{1,2}$ Fawaad Iqbal, MD, ${ }^{3}$ Bruce E. Pollock, MD, ${ }^{1,4}$ Michael J. Link, MD, ${ }^{4}$ Kathy Stien, RN, ${ }^{2}$ Yolanda I. Garces, MD, \\ Paul D. Brown, MD, ${ }^{5}$ Robert L. Foote, $\mathrm{MD}^{1 *}$ \\ ${ }^{1}$ Department of Radiation Oncology, Mayo Clinic, Rochester, Minnesota, ${ }^{2}$ Department of Radiation Oncology, University of Michigan, Ann Arbor, Michigan, \\ ${ }^{3}$ RS McLaughlin Durham Regional Cancer Center, Oshawa, Ontario, Canada, ${ }^{4}$ Department of Neurosurgery, Mayo Clinic, Rochester, Minnesota, \\ ${ }^{5}$ Department of Radiation Oncology, MD Anderson Cancer Centre, Houston, Texas. \\ Accepted 4 June 2014 \\ Published online 1 August 2014 in Wiley Online Library (wileyonlinelibrary.com). DOl 10.1002/hed.23798
}

\begin{abstract}
Background. Stereotactic radiosurgery is widely applied to deliver additional dose to head and neck tumors. However, its safety and efficacy remains equivocal.

Methods. One hundred eighty-four patients with primary head and neck cancers treated between January 1990 and August 2012 with Gamma Knife stereotactic radiosurgery were retrospectively reviewed.

Results. Two hundred fifteen sites were treated with Gamma Knife stereotactic radiosurgery among 184 patients. Fifty-one percent of patients received concurrent external beam radiotherapy (EBRT), 72\% had prior surgery, and $46 \%$ received chemotherapy. Most (44\%) had squamous cell carcinoma and most patients (65\%) were treated for recurrent dis-
\end{abstract}

ease. With a median follow-up of 17.3 months, 12-month local control was $82 \%$. Late effects occurred in 59 patients with the most common being temporal lobe necrosis (15 patients).

Conclusion. Radiosurgery can provide tumor control for patients with head and neck cancers involving the skull base. Long-term follow-up is important in survivors to identify late effects. (c) 2014 Wiley Periodicals, Inc. Head Neck 37: 1557-1562, 2015

KEY WORDS: head and neck malignancy, stereotactic radiosurgery, late effects, boost, chemoradiation

\section{INTRODUCTION}

Stereotactic radiosurgery has been applied to head and neck cancers in the setting of multimodality treatment, including primary radiotherapy of residual disease postoperatively or as part of primary chemoradiotherapy. It has also been used as salvage treatment for recurrence after definitive treatment. However, long-term outcomes are not well defined for stereotactic radiosurgery in the head and neck cancer population.

Even after aggressive initial therapy, local recurrence is a major issue for primary locally advanced head and neck cancers. Radiosurgery offers the ability to dose escalate in sensitive areas, especially at the base of the skull. This is an attractive option because stereotactic radiosurgery can be delivered with great precision and conformality to the site in question. With the addition of stereotactic radiosurgery to the treatment of patients who have already undergone multiple modalities, including surgery, chemotherapy, and external beam radiotherapy (EBRT), the potential for side effects may also increase. The benefit of stereotactic radiosurgery needs to be balanced with the potential for toxicity.

*Corresponding author: R. Foote, Department of Radiation Oncology, Mayo Clinic, 200 First Street SW, Rochester, MN 55905.

E-mail: foote.robert@mayo.edu

\section{MATERIALS AND METHODS}

Between January 1, 1990, and August 1, 2012, 375 patients were treated in the head and neck region with Gamma Knife stereotactic radiosurgery at the Mayo Clinic. This population excluded all patients who were treated for intracranial parenchymal brain disease. Only patients with head and neck cancers (184 patients with nasopharynx, nasal cavity/paranasal sinuses, oral cavity, oropharynx, hypopharynx, larynx, salivary gland, and skin) were retrospectively reviewed. Information was collected on patient age, sex, histology, stereotactic radiosurgery site treated, tumor grade, treatment intent, primary versus recurrent versus metastatic disease, concurrent and prior EBRT, and chemotherapy treatment. Tumor staging was not consistently collected for most patients as many were referred from outside institutions. This was especially true for patients who presented with recurrent disease and had their primary treatment and initial staging performed at an outside institution. Local control, distant progression, radiographic response to treatment, and late toxicity were also reviewed. Gamma Knife stereotactic radiosurgery parameters were recorded for each patient, including marginal dose (minimum tumor dose), isodose prescription line, maximum diameter treated, treatment volume, number of isocenters treated, and maximum dose.

Descriptive statistics were performed using JMP software version 9.01 (SAS Institute, Cary, NC). Median 
progression-free survival, overall survival, and follow-up from the date of Gamma Knife stereotactic radiosurgery treatment were also calculated. Progression-free survival was defined as any progression (local, regional 'nodal', or distant) from the date of Gamma Knife stereotactic radiosurgery treatment. Local failure was defined as in-field progression over serial imaging with CT or MRI. This study was approved by the Mayo Clinic Institutional Review Board. In compliance with Minnesota State statutes, all living patients consented to the review of their medical records for research purposes.

Although most data were available in a prospectively collected Mayo Clinic stereotactic radiosurgery database, all data were verified by retrospective medical chart review. Additional information was gathered from the electronic medical records for follow-up visits and surveillance imaging. The recording of late effects was based on physician assessment and documentation in the electronic medical record. The definition of late toxicity was any adverse event related to the stereotactic radiosurgery treatment site (side effects attributed to EBRT outside of the stereotactic radiosurgery site were excluded). Adverse events were graded using the Common Terminology Criteria for Adverse Events version 4.0 (National Cancer Institute, published June 14, 2010).

Our general Gamma Knife stereotactic radiosurgery treatment entailed patients being consented for stereotactic radiosurgery as same-day out-patient surgery. Patients presented for placement of a rigid head frame under local anesthetic by neurosurgery. Usual practice involved IV placement, sublingual Ativan $1 \mathrm{mg}$ before head frame placement, and a single dose of dexamethasone $8 \mathrm{mg} \mathrm{PO} /$ IV immediately after Gamma Knife stereotactic radiosurgery. With frames in place, fine-cut gadolinium enhanced MRIs (1 mm slice T1 sequence) were performed through the region of interest, transferred to Leksell GammaPlan and used for treatment planning. In patients where tumors were close to the cochlea or optic structures, fine slice CT scans were also performed and fused with the planning MRI. Patients were observed for 2 hours poststereotactic radiosurgery and then discharged. No patients required overnight hospital stays. Patients who were receiving Gamma Knife stereotactic radiosurgery as a boost in addition to EBRT had their external beam treatments held for that day.

\section{RESULTS}

\section{Patient demographics}

A total of 215 sites were treated with Gamma Knife stereotactic radiosurgery in 184 patients during the study period (Table 1). Most patients were men (115 of 184; $63 \%)$ and the majority was treated for head and neck squamous cell carcinoma (81 of $184 ; 44 \%$ ). Most sites treated with stereotactic radiosurgery were for recurrent disease $(139$ of $215 ; 65 \%)$ with stereotactic radiosurgery used as a boost in addition to EBRT (105 of 215; 49\%). The timing of the Gamma Knife stereotactic radiosurgery boost was mostly immediately before or early in the EBRT course (first 1-4 weeks; 82 of 109; 75\%). More than $70 \%$ of patients had head and neck surgeries before their stereotactic radiosurgery treatments (133 of 184; $72 \%$ ). The median EBRT dose was 61.2 Gy (range, 1875.2 Gy), mostly delivered on a daily basis (median 33 fractions; range, 15-58 fractions). The most common sites treated with Gamma Knife stereotactic radiosurgery were the cavernous sinus (52 of $215 ; 24 \%)$ and the skull base (47 of $215 ; 22 \%$ ). Nearly half the patients received concurrent chemotherapy with EBRT (85 of 184; 46\%). Sixteen percent of patients (30 of 184) had documentation of prior EBRT.

\section{Stereotactic radiosurgery dose}

Most treatments were prescribed to the $50 \%$ isodose line with a median dose of 14 Gy (Table 2). The median longest diameter treated was $45.3 \mathrm{~mm}$ and the median maximum stereotactic radiosurgery dose $(100 \%$ isodose line) was $30 \mathrm{~Gy}$. The median tumor volume was $15.9 \mathrm{cc}$ (range, 5.9-175.9 cc). A median of 9 isocenters were used to treat the tumor volumes. There was no influence of treatment intent on Gamma Knife stereotactic radiosurgery parameters, so these have not been reported separately.

\section{Local control}

Median follow-up from the date of stereotactic radiosurgery treatment was 17.3 months (range, 0.1-46 months). Local control at 12 months was $82 \%$ (see Figures 1C and 1D for an example of sustained successful treatment), although 56 patients did eventually experience local failure and 15 patients experienced marginal failure (see Figures 1A and 1B for an example of marginal failure). Local control was statistically different (log-rank $p=.002$ ) among patients stratified by treatment type and intent (EBRT + stereotactic radiosurgery boost, salvage stereotactic radiosurgery alone with curative intent, and palliative stereotactic radiosurgery alone). EBRT + stereotactic radiosurgery boost had the best local control $(88 \%$ at 12 months) compared with stereotactic radiosurgery salvage alone and palliative stereotactic radiosurgery. There was no difference in 12-month local control between salvage stereotactic radiosurgery alone with curative intent and palliative stereotactic radiosurgery alone ( $73 \%$ vs $75 \%$, respectively). In the patients who received $\mathrm{EBRT}+$ stereotactic radiosurgery boost, there was no statistically significant difference in local control when stratified by the timing of stereotactic radiosurgery boost (before, during, or after EBRT; local control at 12 months ranged from $75 \%$ to $92 \%$, but the number of patients was small in each group, likely accounting for the lack of statistical power). The use of chemotherapy had no effect on local control $(p=.83)$. Paradoxically, patients who had had prior surgery had poorer local control at 1 year ( $p=.013 ; 78 \%$ vs $83 \%$ at 1 year).

Of the 215 sites treated, 23 marginal failures occurred (Table 3). Most marginal failures occurred in patients who had recurrent disease (20 of 23 patients) who received palliative stereotactic radiosurgery alone (12 of 23 patients). Sites of failure were most commonly in the bony sinuses (ethmoid, maxillary) and in the cavernous sinus (12 of 23). 
TABLE 1. Patient demographics.

\begin{tabular}{|c|c|}
\hline Sex & $N=184$ \\
\hline Male & 115 \\
\hline Female & 69 \\
\hline $\begin{array}{l}\text { Median age at stereotactic } \\
\text { radiosurgery in years }\end{array}$ & $\begin{array}{l}62 \text { (range, } \\
25-98)\end{array}$ \\
\hline Histology & $N=184$ \\
\hline Squamous cell carcinoma & 81 \\
\hline Salivary carcinoma & 50 \\
\hline Nasopharyngeal carcinoma & 26 \\
\hline Esthesioneuroblastoma & 13 \\
\hline $\begin{array}{l}\text { Other (sarcoma, neuroendocrine, } \\
\text { basal cell carcinoma) }\end{array}$ & 14 \\
\hline Grade & $N=184$ \\
\hline $\begin{array}{l}\text { High/intermediate/low/unknown } \\
\text { or not applicable }\end{array}$ & $61 / 16 / 13 / 94$ \\
\hline Local disease status & $N=215$ \\
\hline Primary & 67 \\
\hline Recurrent & 148 \\
\hline Intent of stereotactic radiosurgery treatment & $N=215$ \\
\hline Boost with EBRT & 109 \\
\hline $\begin{array}{l}\text { Palliative stereotactic radiosurgery alone } \\
\text { (for symptom relief alone) }\end{array}$ & 63 \\
\hline $\begin{array}{l}\text { Salvage stereotactic radiosurgery alone } \\
\text { (with curative intent) }\end{array}$ & 43 \\
\hline Stereotactic radiosurgery timing relative to EBRT & $N=109$ \\
\hline Stereotactic radiosurgery before EBRT course & 41 \\
\hline Stereotactic radiosurgery during EBRT course & 41 \\
\hline Stereotactic radiosurgery after EBRT course & 21 \\
\hline Unknown & 6 \\
\hline Median EBRT prescription dose & $N=186$ \\
\hline Dose & $\begin{array}{l}\text { 61.2 Gy (range, } \\
18-75.2 \text { Gy) }\end{array}$ \\
\hline $\begin{array}{l}\text { Repeat EBRT at time of stereotactic radiosurgery, } \\
\text { prior radical treatment }\end{array}$ & $N=215$ \\
\hline Yes & 30 \\
\hline No & 98 \\
\hline Unknown & 87 \\
\hline Prior head and neck surgery & $N=184$ \\
\hline Yes & 133 \\
\hline No & 51 \\
\hline Chemotherapy & $N=184$ \\
\hline Concurrent & 85 \\
\hline Palliative & 6 \\
\hline Adjuvant & 2 \\
\hline None & 57 \\
\hline Unknown & 34 \\
\hline \multicolumn{2}{|l|}{$\begin{array}{l}\text { Mean stereotactic radiosurgery dose } \\
\text { based on treatment intent }\end{array}$} \\
\hline Boost with EBRT & $\begin{array}{l}11.4 \text { Gy (range, } \\
8-18 \mathrm{~Gy} \text { ) }\end{array}$ \\
\hline $\begin{array}{l}\text { Palliative stereotactic radiosurgery alone } \\
\text { for symptom relief alone }\end{array}$ & $\begin{array}{l}14.3 \text { Gy (range, } \\
10-18 \text { Gy) }\end{array}$ \\
\hline $\begin{array}{l}\text { Salvage stereotactic radiosurgery alone } \\
\text { (with curative intent) }\end{array}$ & $\begin{array}{l}15 \text { Gy (range, } \\
10-20 \mathrm{~Gy})\end{array}$ \\
\hline Location treated with stereotactic radiosurgery & $N=215$ \\
\hline Cavernous sinus & 52 \\
\hline Meckel's cave & 10 \\
\hline Clivus & 6 \\
\hline Ethmoid/sphenoid/maxillary sinus & 34 \\
\hline Skull base & 47 \\
\hline Cranial nerve $\mathrm{V}$ & 6 \\
\hline Nasopharynx & 15 \\
\hline $\begin{array}{l}\text { Other (cerebellopontine angle, } \\
\text { pterygopalatine fossa, infraorbital foramen) }\end{array}$ & 45 \\
\hline
\end{tabular}

Abbreviations: EBRT, external beam radiotherapy; Gy, Gray.

\section{Late toxicity}

Sixty-nine events were coded as late effects among 59 patients (Table 4). Median time to the development of late effects was 17.7 months. Late effects were highly varied depending on the anatomic area treated, but the most common ones included radiation necrosis of the brain $(15$ of $69 ; 22 \%)$ and cranial nerve palsies (11 of 69; $16 \%$ ). Only new cranial neuropathies that occurred after stereotactic radiosurgery treatment in the absence of preceding deficits from surgery, prior EBRT, or tumor progression were coded as late toxicities. The most commonly treated areas were the cavernous sinus (17 of 59; $29 \%$ ) and the nasopharynx (12 of 59;20\%). Most late effects were grade 1 and 2 toxicities, mostly asymptomatic cranial neuropathies or radiation necrosis. There were 14 grade 3 and above toxicities. There were 11 grade 3 toxicities, which consisted of 5 cases of resection for symptomatic radiation necrosis, 3 cases of severe osteonecrosis requiring debridement, 2 cases of severe hearing loss requiring cochlear implants, and 1 case of stroke. All grade 4 toxicities (3 of 3 ) were complete blindness secondary to stereotactic radiosurgery. In actuarial terms, the late complication (all events grades 1-4) incidence at 1 year was $12 \%$, at 5 years it was $25 \%$, and by 10 years it was $26 \%$. Most complications occurred within the first 5 years after Gamma Knife stereotactic radiosurgery treatment. However, the risk of grade 3 and above late effects increased significantly beyond 5 years. The actuarial risk of grade 3 and above late effects was only $5 \%$ in the first 12 months but increased to $15 \%$ at 24 months and $40 \%$ by 48 months.

Stereotactic radiosurgery dose parameters, such as marginal dose, tumor volume, or tumor diameter, were not correlated with the development of late toxicity. Stereotactic radiosurgery target site or treated location also had no bearing on late toxicity. The use of chemotherapy $(p<.004)$ and prior surgery $(p<.001)$ were correlated with late toxicity development, but repeat EBRT was not $(p=.20)$.

\section{Survival}

Overall survival from the date of initial diagnosis in all patients was $41 \%$ at 12 months with a median survival of 9 months. Overall survival and progression-free survival from the end of stereotactic radiosurgery treatment were $16 \%$ and $57 \%$ at 12 months, respectively. Median overall survival and progression-free survival post-stereotactic radiosurgery treatment was 3 months and 16 months, respectively. Poor overall survival posttreatment likely reflects the large number of patients who were treated for recurrence and for palliation. When patients were stratified by treatment intent (EBRT + stereotactic radiosurgery boost, salvage stereotactic radiosurgery alone with curative intent, and palliative stereotactic radiosurgery alone), those who received a boost with EBRT had a much higher median progression-free survival (39 months; log-rank $p=.0001)$. Median progressionfree survival postsalvage stereotactic radiosurgery treatment alone with curative intent was 16.1 months, whereas patients treated with palliative stereotactic radiosurgery alone had a median progression-free survival of 8 months. Interestingly, patients treated with salvage stereotactic radiosurgery alone 
TABLE 2. Stereotactic radiosurgery dose characteristics $(N=215)$.

\begin{tabular}{|c|c|}
\hline $\begin{array}{l}\text { Median stereotactic } \\
\text { radiosurgery dose characteristics }\end{array}$ & Value (range) \\
\hline $\begin{array}{l}\text { Maximum stereotactic } \\
\text { radiosurgery dose }\end{array}$ & 30 Gy (24-40 Gy) \\
\hline Volume treated & 15.9 cc $(5.9-175.9 \mathrm{cc})$ \\
\hline $\begin{array}{l}\text { Marginal stereotactic } \\
\text { radiosurgery prescription dose } \\
\text { (minimal tumor dose) }\end{array}$ & 14 Gy (12-20 Gy) \\
\hline Prescription isodose line & $50 \%(25 \%$ to $70 \%)$ \\
\hline Maximum diameter treated & $45.3 \mathrm{~mm}(19.6-90 \mathrm{~mm})$ \\
\hline $\begin{array}{l}\text { Number of stereotactic } \\
\text { radiosurgery isocenters }\end{array}$ & $9(1-22)$ \\
\hline
\end{tabular}

Abbreviation: Gy, Gray.

with curative intent had the best median overall survival (15.2 months; $p=.02$ ), although boost stereotactic radiosurgery was similar at 9.4 months and palliative patients lived a median of 6.6 months post-stereotactic radiosurgery treatment. Histology and chemotherapy use had no impact on overall survival or progression-free survival. Although surgery had no impact on overall survival, prior surgery was correlated with worse progression-free survival $(p=.0001$; $52 \%$ vs $76 \%$ at 1 year).

\section{DISCUSSION}

This study represents one of the largest series of patients with head and neck cancer treated with radiosurgery. Although the patient population is heterogeneous, as were the sites treated, it provides valuable information on the potential late effects of stereotactic radiosurgery treatment in long term survivors. The majority of our patients was referred for treatment of recurrent disease and most received concurrent chemoradiotherapy with a stereotactic radiosurgery boost, although a number did receive curative-intent stereotactic radiosurgery salvage alone. The combination of EBRT and stereotactic radiosurgery boost resulted in a substantial median progression-free survival of nearly 40 months. This is likely also related to volume of disease before treatment and performance status, although treatment intent did not influence the dose or volume of tissue treated with Gamma Knife stereotactic radiosurgery. Our overall survival outcome of $40 \%$ at 12 months is also reflective of our patient population, which included predominantly palliative and recurrent patients.
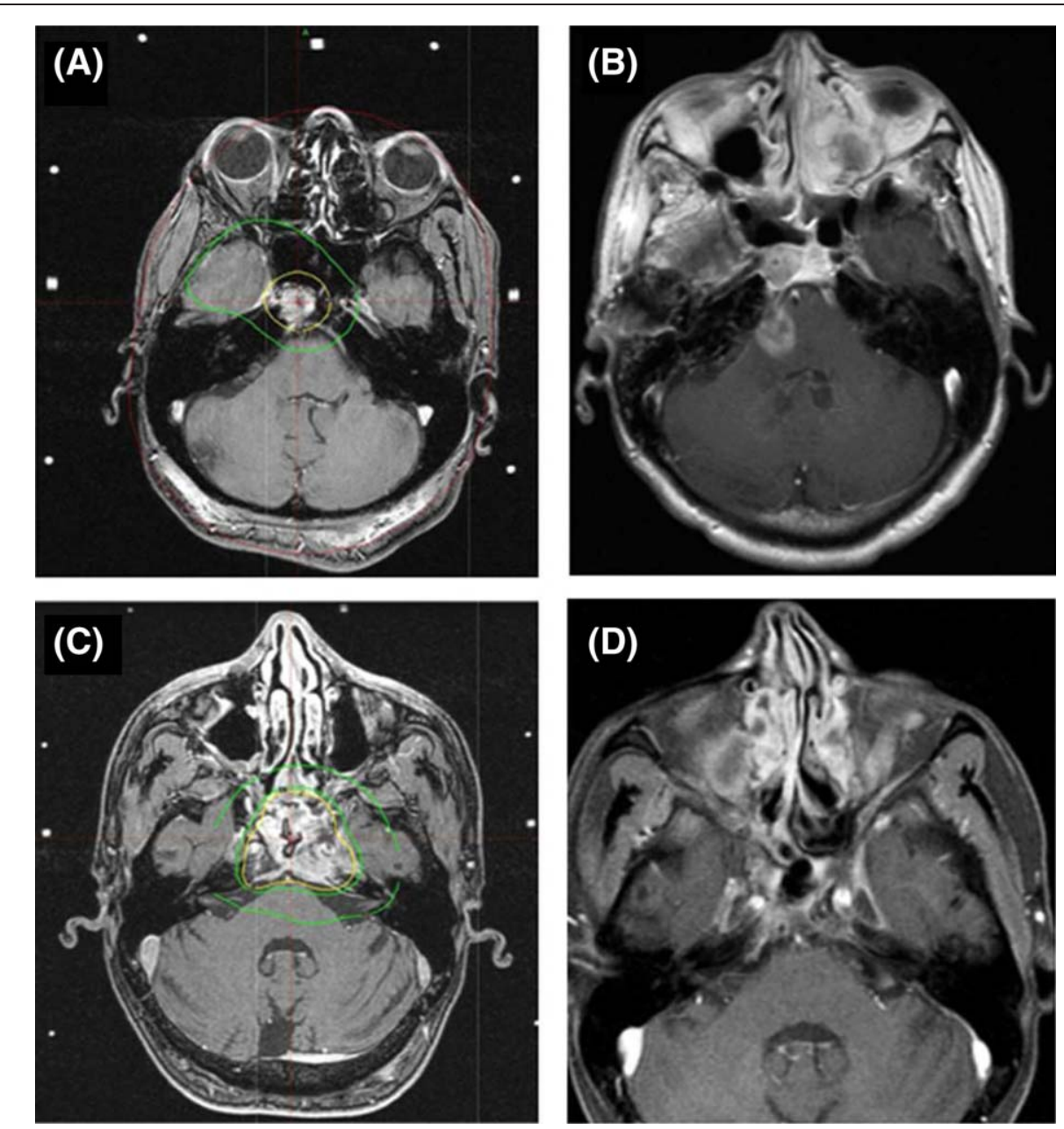

FIGURE 1. (A) Squamous cell carcinoma boost; (B) marginal failure 1 year later but local control of treated disease encompassed by prescription $50 \%$ isodose line (yellow) remained intact; (C) adenocarcinoma boost in a different patient; and (D) local control 3 years later. 
TABLE 3. Characteristics of marginal failures.

\begin{tabular}{lc}
\hline Location treated with Gamma Knife & $N=23$ \\
Cavernous sinus & 5 \\
Meckel's cave & 3 \\
Ethmoid/sphenoid/maxillary sinus & 7 \\
Skull base & 3 \\
Cranial nerve $V$ & 1 \\
Other (cerebellopontine angle, orbital fossa) & $4=23$ \\
Histology & 8 \\
Squamous cell carcinoma & 11 \\
Salivary cancers & 4 \\
Other (nasopharyngeal carcinoma, & \\
esthesioneuroblastoma, skin cancer) & $N=23$ \\
Recurrent vs primary vs metastatic & 20 \\
Recurrent & 1 \\
Primary & 2 \\
Metastatic & $N=23$ \\
Intent of treatment & 4 \\
Stereotactic radiosurgery curative & 7 \\
Boost with EBRT & 12 \\
Stereotactic radiosurgery palliative & $14 \mathrm{~Gy}$ (range, \\
Median stereotactic radiosurgery dose & $10-20 \mathrm{~Gy}$ ) \\
& $14.9 \mathrm{cc}$ (range, \\
Median stereotactic radiosurgery & $2.7-85.1 \mathrm{cc}$ ) \\
tumor volume & \\
\hline
\end{tabular}

Abbreviations: EBRT, external beam radiotherapy; Gy, Gray.

Most prior studies have focused on particular histologies of head and neck cancers in the primary curative treatment setting (initial diagnosis) as well as in the recurrent setting. Many of these studies demonstrate good local control but with a moderate risk of cranial nerve neuropathy and brain radionecrosis. Long-term follow-up is essential for delineating late effects.

Firlik et al $^{1}$ reported one of the first series of radiosurgery for recurrent head and neck cancers after prior surgery and EBRT. Although follow-up was highly varied for the 12 patients in this series (range, 3-35 months), no late effects were documented, but the authors noted that 11 of 12 patients had cranial nerve deficits before stereotactic radiosurgery salvage. Local control was reported as a crude rate of 8 of 12 patients based on imaging characteristics. ${ }^{1}$

Stereotactic radiosurgery boost has been reported for the treatment of salivary tumors both in the curative primary setting and in the recurrent setting. Douglas et $\mathrm{al}^{2}$ published a series of 34 patients with salivary cancer treated with a planned Gamma Knife stereotactic radiosurgery boost after neutron radiotherapy. Forty-month local control was significantly better in boosted patients compared to historical controls who had received only neutron radiotherapy, $82 \%$ versus $39 \%(p=.04)$. At a median follow-up of 20.5 months, 1 patient had died because of an infection. Complications were similar whether tumors were boosted or not, with most side effects due to the neutron beam therapy (xerostomia, trismus, skin fibrosis). Three patients developed radiation necrosis. ${ }^{2}$ Lee et $\mathrm{al}^{3}$ reviewed a series of 8 patients with 16 salivary tumor skull base recurrences. Allowing for salvage stereotactic radiosurgery, 2-year local freedom from progression was $75 \%$. Once again, radiation necrosis was a documented complication (in this series, 1 of 8 patients). Similarly, a study from Japan examining 12 patients with recurrent adenoid cystic carcinoma at the
TABLE 4. Late toxicity (69 events in 59 patients).

\begin{tabular}{|c|c|}
\hline Median time to late toxicity & $\begin{array}{r}17.7 \mathrm{mo} \text { (range, } \\
0.9-127.8 \mathrm{mo})\end{array}$ \\
\hline Sex & $N=59$ \\
\hline Male & 37 \\
\hline Female & 22 \\
\hline Histology & $N=59$ \\
\hline Squamous cell carcinoma & 26 \\
\hline Salivary carcinoma & 15 \\
\hline Nasopharyngeal carcinoma & 12 \\
\hline Esthesioneuroblastoma & 4 \\
\hline $\begin{array}{l}\text { Other (neuroendocrine, } \\
\text { basal cell carcinoma) }\end{array}$ & 2 \\
\hline Location treated with Gamma Knife & $N=59$ \\
\hline Cavernous sinus & 17 \\
\hline Meckel's cave & 1 \\
\hline Clivus & 2 \\
\hline Ethmoid/sphenoid/maxillary sinus & 7 \\
\hline Skull base & 8 \\
\hline Cranial nerve $\mathrm{V}$ & 4 \\
\hline Nasopharynx & 12 \\
\hline $\begin{array}{l}\text { Other (CPA, pterygopalatine } \\
\text { fossa, infraorbital foramen) }\end{array}$ & 8 \\
\hline Recurrent vs primary vs metastatic & $N=69$ \\
\hline Recurrent & 35 \\
\hline Primary & 31 \\
\hline Metastatic & 3 \\
\hline Intent of treatment & $N=69$ \\
\hline Stereotactic radiosurgery curative & 15 \\
\hline Boost with EBRT & 45 \\
\hline Stereotactic radiosurgery palliative & 9 \\
\hline Type of toxicity & $N=69$ \\
\hline Xerostomia & 4 \\
\hline Vocal cord paralysis & 1 \\
\hline Stroke & 1 \\
\hline Radiation necrosis (brain) & 15 \\
\hline Ptosis & 2 \\
\hline Pain & 7 \\
\hline Infection & 5 \\
\hline Endocrine abnormalities & 2 \\
\hline Hearing loss & 3 \\
\hline Fistula & 2 \\
\hline Fibrosis & 1 \\
\hline Facial numbness & 8 \\
\hline Eustachian tube dysfunction & 1 \\
\hline Dysphagia & 1 \\
\hline Decreased vision & 5 \\
\hline Cranial nerve palsy & 11 \\
\hline \multicolumn{2}{|l|}{ CTCAE grading of late toxicity } \\
\hline 1 & 24 \\
\hline 2 & 31 \\
\hline 3 & 11 \\
\hline 4 & 3 \\
\hline
\end{tabular}

Abbreviations: CPA, cerebellopontine angle; EBRT, external beam radiotherapy; CTCAE, Common Terminology Criteria for Adverse Events.

skull base reported crude local control rates of 10 of 12 patients with a median overall survival of 18 months. However, there was no discussion of late effects. ${ }^{4}$

Stereotactic radiosurgery has been studied extensively in the primary treatment of nasopharyngeal cancers. Multiple retrospective studies have demonstrated improved local control ranging from $93 \%$ to $100 \%$ at 3 years for stereotactic radiosurgery boost after radical EBRT (series had doses ranging from 64.8-66 Gy) with concurrent 
chemotherapy. ${ }^{5-8}$ Most series noted minimal late effects after treatment, although follow-up ranged from 12 to 21 months. In a recent series from Stanford detailing such treatment, late toxicity was documented in 14 of 82 patients, with the most common complication being radiation necrosis (10 of 14 patients). ${ }^{6}$

Stereotactic radiosurgery also seems to be a good option for recurrent nasopharyngeal cancer involving the base of the skull. One series of 36 patients treated for recurrent disease with EBRT (range, 20-60 Gy) followed by stereotactic radiosurgery boost (range, 8-20 Gy) reported a 3-year local control rate of $56 \%$ and a 5-year OS of $49 \%$. ${ }^{9}$ Eleven percent developed nasopharyngeal necrosis, and 1 patient developed asymptomatic skull base destruction. ${ }^{9}$ Another series of 47 patients with base of skull lesions, including nasopharyngeal cancer, examined the use of stereotactic radiosurgery alone for salvage (median stereotactic radiosurgery dose $12 \mathrm{~Gy}$ ). ${ }^{10} \mathrm{~A}$ crude local control rate of $69 \%$ was achieved at a median follow-up of 9 months. Three of 59 patients developed cranial nerve neuropathies. ${ }^{10}$ Another series of 10 patients showed a local control of $100 \%$ with only 1 patient developing a late effect (new cranial neuropathy in the absence of disease progression). ${ }^{11}$

Given these heterogeneous reports, our local control rate of $82 \%$ is quite comparable to the published literature. In our series, the most common late effects were radiation necrosis of the brain and cranial nerve deficits. These accounted for more than $20 \%$ of all late effects. Most late complications occurred within the first 5 years of treatment (5-year complication incidence of $26 \%$ on actuarial analysis). The cavernous sinus was the most commonly treated stereotactic radiosurgery site in our series. A number of retrospective series have focused on the treatment of these lesions and the rate of cranial neuropathy has ranged from $0 \%$ to $10 \% .^{12-14}$ However, the follow-up in these series has been quite short $(<14$ months). In our series, the median time to develop late effects was 17.7 months. Our study has reasonable longterm follow-up, such that we documented 59 patients of 184 developing late effects (crude complication rate of $32 \%$ ). Our higher complication rate likely reflects the effects of recurrent disease and greater morbidity with prior treatment and trimodality salvage treatment (most patients in our series received concurrent chemoradiotherapy and stereotactic radiosurgery). Indeed, this is reflected by the correlation of prior surgery and chemotherapy use with late effects. Our findings that surgery was negatively associated with local control and progression-free survival is paradoxical, but likely reflects difficult to control recurrent disease that was initially treated with surgery alone and which was then referred to the Mayo Clinic, for salvage chemoradiation and stereotactic radiosurgery boost.

Our study was limited by its retrospective nature. Key issues to correlating predictive factors for late effects, such as prior radiotherapy and radiotherapy dose, were not easily addressed, especially if patients were treated many years ago (early 1990s) before referral to our institution. This missing information impeded our ability to calculate cumulative doses to normal structures. The lack of accurate staging information of the initial primary tumor was also a major limitation. Palliation of symptoms with stereotactic radiosurgery was also not welldocumented and was excluded from our primary analysis.

In spite of these shortcomings, this study is valuable in evaluating the long-term outcome as well as feasibility of stereotactic radiosurgery in the setting of head and neck cancers. Stereotactic radiosurgery is a well-tolerated procedure that provides good local tumor control, especially for patients who are able to receive it as a boost in addition to chemoradiotherapy. Even in the salvage setting using stereotactic radiosurgery alone, it seems to provide good local control. However, this needs to be balanced with long-term follow-up and evaluation of concurrent or prior radiotherapy treatments as cranial nerve neuropathies and radiation necrosis were fairly common complications of such treatment.

\section{REFERENCES}

1. Firlik KS, Kondziolka D, Lunsford LD, Janecka IP, Flickinger JC. Radiosurgery for recurrent cranial base cancer arising from the head and neck. Head Neck 1996;18:160-165; discussion 166.

2. Douglas JG, Goodkin R, Laramore GE. Gamma knife stereotactic radiosurgery for salivary gland neoplasms with base of skull invasion following neutron radiotherapy. Head Neck 2008;30:492-496.

3. Lee N, Millender LE, Larson DA, et al. Gamma knife radiosurgery for recurrent salivary gland malignancies involving the base of skull. Head Neck 2003;25:210-216.

4. Mori Y, Kobayashi T, Kida Y, Oda K, Shibamoto Y, Yoshida J. Stereotactic radiosurgery as a salvage treatment for recurrent skull base adenoid cystic carcinoma. Stereotact Funct Neurosurg 2005;83:202-207.

5. Le QT, Tate D, Koong A, et al. Improved local control with stereotactic radiosurgical boost in patients with nasopharyngeal carcinoma. Int J Radiat Oncol Biol Phys 2003;56:1046-1054.

6. Hara W, Loo BW Jr, Goffinet DR, et al. Excellent local control with stereotactic radiotherapy boost after external beam radiotherapy in patients with nasopharyngeal carcinoma. Int $J$ Radiat Oncol Biol Phys 2008;71:393-400.

7. Chen HH, Tsai ST, Wang MS, et al. Experience in fractionated stereotactic body radiation therapy boost for newly diagnosed nasopharyngeal carcinoma. Int J Radiat Oncol Biol Phys 2006;66:1408-1414.

8. Tate DJ, Adler JR Jr, Chang SD, et al. Stereotactic radiosurgical boost following radiotherapy in primary nasopharyngeal carcinoma: impact on local control. Int J Radiat Oncol Biol Phys 1999;45:915-921.

9. Pai PC, Chuang CC, Wei KC, Tsang NM, Tseng CK, Chang CN. Stereotactic radiosurgery for locally recurrent nasopharyngeal carcinoma. Head Neck 2002;24:748-753.

10. Cmelak AJ, Cox RS, Adler JR, Fee WE Jr, Goffinet DR. Radiosurgery for skull base malignancies and nasopharyngeal carcinoma. Int J Radiat Oncol Biol Phys 1997:37:997-1003.

11. Chua DT, Sham JS, Hung KN, Kwong DL, Kwong PW, Leung LH. Stereotactic radiosurgery as a salvage treatment for locally persistent and recurrent nasopharyngeal carcinoma. Head Neck 1999;21:620-626.

12. Yamamoto M, Kida Y, Fukuoka S, et al. Gamma Knife radiosurgery for hemangiomas of the cavernous sinus: a seven-institute study in Japan. $J$ Neurosurg 2010;112:772-779.

13. Iwai Y, Yamanaka K, Yoshimura M. Gamma knife radiosurgery for cavernous sinus metastases and invasion. Surg Neurol 2005;64:406-410; discussion 410 .

14. Kano H, Niranjan A, Kondziolka D, Flickinger JC, Lunsford LD. The role of palliative radiosurgery when cancer invades the cavernous sinus. Int $J$ Radiat Oncol Biol Phys 2009;73:709-715. 